\title{
La estructura conceptual de los miedos urbanos*
}

\section{The conceptual structure of urban fears}

\author{
Pablo Páramo** \\ Universidad Pedagógica Nacional, \\ Colombia \\ Edgar Roa \\ Universidad Piloto de Colombia \\ Recibido: 2 de febrero de 2015 \\ Revisado: 3 de marzo de 2015 \\ Aceptado: 30 de abril de 2015
}

\section{Resumen}

Este trabajo indaga por los temores que experimentan los habitantes de las ciudades y por la estructura conceptual que comparten sobre las situaciones temidas actualmente. Se analizan los resultados de 130 entrevistas semiestructuradas, orientadas a identificar los temores más comunes de hombres y mujeres asociados a la ciudad y la estructura conceptual que subyace a estos temores. Los resultados obtenidos se analizaron con un Escalamiento Multidimensional, que permitió identificar la estructura del miedo que muestran los participantes a distintas situaciones. Se proponen acciones en procura de la reducción de la percepción de inseguridad en la ciudad.

Palabras clave: miedo al crimen, psicología (criminal) crímenes en la calle, reacciones al crimen (fuente: Tesauro UNICRI).

\section{Abstract}

This paper focuses on fears experienced by the dwellers of the city and the conceptual structure shared on the situations that currently are feared. The results of 130 semi-structured interviews aimed at identifying the most common fears of men and 
women associated with the city and the conceptual structure underlying these fears are analyzed. The results were analyzed trough a Multidimensional Scaling, which identified the conceptual fear structure of participants to different situations. Actions in an attempt to reduce the perception of insecurity in the city are proposed.

Keywords: Crime (fear of), psychology (criminal), Street crimes, reactions to crime (fuente: Tesauro UNICRI)

\section{Introducción}

El presente trabajo tiene como propósito estudiar el miedo en las ciudades y, particularmente, las representaciones de estos temores en sus habitantes. El análisis parte del papel que han jugado los medios en la construcción social del miedo a la ciudad en épocas recientes y del tipo de mediciones que se suelen hacer para evaluar dicho aspecto. Más adelante se justifica la importancia del estudio y se describe la metodología utilizada, la cual se fundamentó en entrevistas individuales a hombres y mujeres, mediante las cuales se logra caracterizar los aspectos temidos por los participantes del estudio y la estructura conceptual de estos temores.

\section{Descripción general del problema}

Los miedos o temores han afectado desde siempre la vida en las ciudades. En la actualidad, esta respuesta emocional afecta las relaciones sociales de tal manera que el temor al otro y a salir a la calle se está convirtiendo en una conducta generalizada que afecta considerablemente la calidad de vida urbana en buena parte de las ciudades del mundo. Los nuevos miedos (al terrorismo, al cambio climático, a las confrontaciones religiosas, a la culturización, a los inmigrantes, a las catástrofes naturales, a las epidemias, a los asaltos callejeros, etc.) están afectando la convivencia entre las culturas del mundo entero.

A las altas tasas de homicidios $\mathrm{y}$, en general, a la criminalidad se suma el "discurso del terror" con el que los medios contribuyen a la construcción social del miedo. Si bien existen los peligros reales, que se evidencian de forma objetiva en las tasas de homicidios, robos y violaciones, entre otras acciones, hay importantes elementos subjetivos que se construyen por influencia psicológica de los medios, con lo que se coadyuva a aumentar el temor, dando lugar a un miedo construido en la ciudad y también a una ciudad construida por el miedo (Cuevas y Gómez, 2014). El poder que tiene el índice de audiencia de los canales de televisión, por ejemplo, en la decisión a la hora de trasmitir noticias, sumado al interés que los ciudadanos muestran por los informes amarillistas, sensacionalistas, sangrientos, presentados día a día y repetidos en la mañana, medio día y noche, que resaltan lo peligrosa que es la ciudad han contribuido a crear una cultura de miedo (Altheide y Gruyter, 2002; Soyinka, 2007; Macek, 2006; Linke y Smith, 2009; Carro, Valera y Vidal, 2010; Valera, Castrechini y Di Masso, 2013).

Tal vez la mayor expansión del fenómeno a través de los medios se vivió a partir del 11-S1, cuando los miedos en las ciudades tuvieron un cambio: del interiorismo de la ciudad y sus propios problemas al exteriorismo de ellas; surge en todo el mundo el miedo a lo cultural y al extranjero, como se vivió en la época de la Edad Media con la peste bubónica, pero en esta ocasión, con el poder de romper culturas.

Estos miedos, que pasan de ser puntuales o que se desencadenan en un territorio específico, se han convertido en un componente global, caracterizados por la ruptura social con el refuerzo de los diversos medios de comunicación (Gutiérrez, 2005). A partir de este acontecimiento, muchos autores han descrito los nuevos miedos que afrontan las ciudades (Borja, 2005, 2008; Bru y Vicente, 2005; Portal y Díaz, 2001; Fraile, Bonastra, Rodríguez y Arella, 2010; Carrion, 2007), quienes han desarrollado un

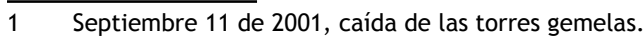


discurso donde aparecen conceptos como la ciudad fortaleza, privatopía, agorafobia y ciudad dual.

Como consecuencia de las altas tasas de criminalidad y de quienes explotan estos temores (las campañas políticas, para elegir a los gobernantes; los constructores, para vender sus viviendas en comunidades cerradas; los medios, para llamar la atención, y las empresas de vigilancia, para que sean contratadas), los habitantes de la ciudad toman decisiones que les permiten tener algún grado de seguridad y reducir sus temores. Dentro de estas acciones se observa el cerramiento de las calles para evitar el ingreso de vehículos o personas desconocidas; la contratación de seguridad privada; la vigilancia -ya sea esta por cámaras, perros etc.-; la privatización de los espacios públicos; la preferencia por los centros comerciales para hacer las compras o transacciones comerciales; la elección de condominios o conjuntos habitacionales cerrados para vivir; la preferencia por centros recreativos privados; y la preferencia por los sociolugares para sociabilizar (Páramo, 2011), con el consecuente abandono de los parques, plazas y demás lugares públicos que solían frecuentarse en el pasado (Páramo y Cuervo, 2009; 2013).

De parte de los gobiernos, las acciones que se toman para disminuir el delito, algunas veces eficaces, no logran cambiar la percepción de temor al salir a la calle, lo cual trae como consecuencia el decaimiento de los espacios públicos al convertirlos en lugares que infunden temor en el ciudadano, y las acciones no resultan lo suficientemente efectivas para lograr revertir el fenómeno.

¿Cómo cambiar esta inconsistencia entre los resultados objetivos que muestran reducción del crimen y los subjetivos, que muestran que la gente vive temerosa? ¿Qué tipo de acciones debería implementarse en la gestión urbana para que, además de reducir la criminalidad, se pueda disminuir el temor a la ciudad? Para tomar decisiones al respecto, un punto de partida podría ser investigar la manera como las personas estructuran sus temores a la ciudad. Este estudio presenta de manera exploratoria cómo estructuran las personas sus temores a las distintas situaciones que se experimentan en el ambiente urbano.

\section{La medición del miedo en las ciudades}

La medición del miedo en las ciudades ha tenido algunos hitos importantes en la historia. Según Morquecho y Vizcarra (2008), en 1730 aparecen las primeras encuestas relacionadas con la victimización en ciudades danesas, y en Francia, para la misma época, con las encuestas que permitían conocer y cuantificar la experiencia de los ciudadanos al momento de un crimen. En Estados Unidos aparece la Encuesta Internacional sobre Criminalidad y Victimización, en 1965, seguida por las de Ferraro y La Grange (1987) y Hale (1996).

Existe actualmente la Encuesta Social General (GBS, por sus siglas en inglés), la cual ha venido haciendo seguimiento al miedo de los estadounidenses desde comienzos de los años setenta del siglo pasado. Por su parte, en Europa, se ha trabajado un instrumento desarrollado por Jackson (2002) y Farrall (2007), que incluye entrevistas en profundidad y análisis estadísticos para las dos fuentes de información, cuantitativas y cualitativas.

Al indagar por estudios más específicos obre el miedo experimentado en los espacios públicos, se observa un interés de los investigadores por explorar variables personales, sociales y ambientales en relación con la percepción de inseguridad. En estos se evidencia que se trata de un fenómeno complejo que involucra, además de algunas variables personales (género, edad, etc.), aquellas asociadas con las representaciones sociales del espacio y la percepción de disponibilidad de apoyo y la evaluación de la calidad ambiental en términos de factores de riesgo, al valorar los alrededores del lugar de vivienda (Carro, Valera y Vidal, 2010).

El estudio de Russo, Roccato y Vieno (2013) en Italia indagó por el papel de las variables individuales (victimización directa o indirecta) a escala de variables personales -como el ser mujer, viejo, con bajo nivel educacional, o habitante de un área residencial con distintas densidades- $y$ en el ámbito ecológico, por lo que los autores quieren significar la tasa de criminalidad de la localidad en la que viven los habitantes, la tasa de desempleo y la tasa de inmigración. 
Como resultado de este cruce de variables se observa que la victimización directa o indirecta -ser mujer, anciano, vivir en una gran ciudad y en un contexto caracterizado por altas tasas de criminalidad y de desempleo- está asociada a la percepción de riesgo de crimen. Hawdon, Räsänen, Oksanen y Vuori (2013), estudiaron recientemente cómo el miedo a diferentes tipos de crímenes influencia la solidaridad de la comunidad, por ejemplo cuando se trata de asesinatos en escuelas donde hay varias víctimas.

Para Latinoamérica y el Caribe se cuenta con el Índice de Ley y Orden que establece la firma encuestadora Gallup. Los datos para 2013 son preocupantes, si se tiene en cuenta que los residentes de esta región son quienes se sienten menos seguros en sus comunidades frente al resto de regiones del mundo: Sur de Asia, Asia Oriental, Estados Unidos y Canadá, Europa y la antigua Unión Soviética. Adicionalmente, al comparar estas percepciones en los últimos años, el estudio muestra que no hay una mejora significativa en los últimos cinco años (Gallup, 2013). En cuanto a una mejor percepción de seguridad, Nicaragua ocupa el primer lugar, Colombia, el séptimo, y Venezuela, el último. En el caso particular de Colombia, se observa una pequeña mejoría en los últimos cinco años.

Por otra parte, al revisar los estudios de miedo desde la perspectiva del género, se evidencia una clara relación entre los miedos de las mujeres y el uso del espacio, lo cual se trata de explicar en términos del sentido de vulnerabilidad física de las mujeres frente a los hombres, particularmente frente al riesgo de una violación o a un asesinato sexual asociado al uso de los espacios públicos y a la forma como estos son planeados (Valentine, 1989; Condon, Lieber y Maillochon, 2007; Action Aid International,, en: http://www.actionaid.org/sites/ files/actionaid/women_and_the_city_ii_1.pdf).

En Colombia y en Bogotá, los estudios relacionados con la percepción del crimen vienen en aumento. El Observatorio de Cultura Urbana, en 1997, con un trabajo denominado Territorios del miedo en Santafé de Bogotá, realizado por Soledad Niño, muestra el tema de la percepción del miedo en la ciudad a partir de los temores más comunes de los bogotanos y de las consecuencias de este temor, como el aislamiento y la disminución de los encuentros sociales (Niño, 1997). Por su parte, el Departamento Nacional de Planeación viene aplicando, desde 2003, una encuesta sobre convivencia y seguridad en los hogares de varias ciudades en Colombia. Entre 2006 y 2007, la Fundación Seguridad y Democracia realiza encuestas sobre criminalidad y victimización en diversas ciudades del país. Igualmente, la Secretaría de Gobierno, la Administración Distrital y el Sistema Unificado de Información de Violencia y Delincuencia de Bogotá (SUIVD) realizaron encuestas en varios hogares sobre la percepción de seguridad. León (2008), recoge algunos estudios que se realizaron en Bogotá, adelantados por entidades gubernamentales y privadas, como la Cámara de Comercio de Bogotá (http://ccb.org.co/contenido/contenido. aspx?cat ID=126\&conID=6574) y la organización Red de Ciudades Cómo vamos (http://www.bogotacomovamos.org/documentos/presentacion-deresultados-comparados-de-la-encues/), en la que se hace seguimiento a las acciones de los gobiernos municipales, dentro de las cuales se evalúa la percepción de seguridad de los ciudadanos.

Desde la aplicación de las encuestas en el siglo xvIII sobre victimización hasta las que se aplican en nuestros días, sobre percepción de inseguridad o de miedo, se exploran por lo general los principales temores que han tenido los habitantes de las ciudades - robo en el hogar, hurto con mano armada y violencia, peleas y riñas callejeras-, y cómo estos temores se asocian con variables personales, sociales y de las características del lugar, principalmente (, Manrique 2006; Bocanegra y Nieto, 2010). Pero lo que no se ha indagado es la manera como se estructuran conceptualmente estos temores en las personas.

\section{La categorización conceptual como fundamento del entendimiento del \\ mundo}

La clasificación de objetos o eventos dentro de categorías o conceptos es una actividad fundamental para poder entender y apropiarnos del mundo en que actuamos. Para sustentar este ar- 
gumento, Hackett (2014) muestra la importancia de las clasificaciones que hacen los individuos para distintas disciplinas científicas. Tanto para la sociología, como para la psicología, la filosofía y las neurociencias, afirma el autor, clasificar es el punto de partida para la comprensión del mundo.

La sociología le da gran importancia al sistema categorial al considerar las influencias de la sociedad sobre cómo los distintos grupos sociales o culturas crean nociones de clase, tiempo y espacio como formas de categorizar sus mundos, a la vez que evidencian las difierencias entre sociedades. En la psicología, la formación de categorías es escencial para explicar procesos básicos como la discriminación y la generalización; se requiere que los estímulos sean entendidos como pertenecientes a una categoría mayor que ellos mismos para que pueda ocurrir la discriminación o generalización. Adicionalmente, en la teoría piagetiana el desarrollo cognoscitivo del niño se da mediante un sistema de clasificación categorial que evoluciona por etapas y estructura el pensamiento.

Kelly, en su teoría de los constructos personales (1991) argumenta que las personas no actúan de forma pasiva en el mundo, sino como científicos, y para ello formulan hipótesis, suponiendo una forma de funcionamiento de las cosas que se pone en contrastación permanentemente. Para ello, la persona clasifica los objetos del mundo que lo rodea y crea categorías para poder hablar de ellos.

Por su parte, en la filosofía se destaca la afirmación de Kant sobre la existencia de categorías a priori que son necesarias para la percepción de todos los posibles objetos y eventos. Todas estas categorías son abstracciones de la realidad y son las que permiten hablar del mundo sin hacer referencia a cada objeto en particular, a la vez que contribuyen a construir la realidad. Y para las neurociencias, las investigaciónes dan evidencia sobre el procesamiento de los estímulos a los que responden los individuos a partir de estructuras neuronales organizadas dentro de categorías (Murphy, 2002). Gracias a ello, los individuos poseen la capacidad de síntesis y de estructurar su entendimiento de mayor complejidad (Pothos y Willis, 2011).
De este modo, las categorías forman la base del entendimiento humano, pero: ¿cómo se relacionan estas categorías entre sí para conseguir una unidad significativa para los individuos? Para el caso que interesa en este estudio: ¿existe alguna estructura categorial o conceptual que les permita a las personas organizar los objetos o situaciones temidas en la ciudad? ¿Hay diferencia en la conceptualización que hacen los hombres respecto de las mujeres frente a los objetos o situaciones temidas? ¿Qué valor podría tener la identificación de esta manera de organizar conceptualmente los temores en la desmitificación de la inseguridad y en la gestión de la seguridad de la ciudad?

\section{Metodología}

El estudio que se resume a continuación es de carácter descriptivo-exploratorio y de investigación básica. Descriptivo, porque se centra en identificar algunos de los miedos más comunes que experimentan los habitantes de los centros urbanos, a la vez que caracteriza la estructura conceptual de dichos temores, y de investigación básica, porque, sin pretender la generalización de los hallazgos, intenta determinar la base conceptual con que las personas estructuran estos temores.

\section{Participantes}

La investigación se fundamenta en la entrevista informal, que se hizo inicialmente de manera individual a 50 personas, para identificar los temores más comunes (Páramo, 2014²), a partir de los cuales se entrevistaron 80 personas más. Todos los consultados estaban en el rango de edad entre 14 y 60 años de edad, $50 \%$ hombres y $50 \%$ mujeres. El muestreo fue de tipo no probabilístico, por conveniencia.

\section{Instrumento}

La información recogida con las primeras 50 personas fue filtrada a partir de la selección de aquellas situaciones que se repitieran más de dos ve-

$\overline{2}$ Investigación realizada a comienzos de 2013. 
ces, lo que dio lugar a una lista de 23 situaciones. Las 23 situaciones más comunes mencionadas por los participantes en el estudio original de Páramo (2014) se escribieron en 23 tarjetas independientes, cada una de 15 centímetros de ancho por 10 centímetros de alto. A este listado se le agregó una más que decía "miedo", conformando así un listado de 24 tarjetas, que se constituyeron en el instrumento para la realización de una entrevista individual basada en el sorteo o clasificación libre de tarjetas (Canter, Brown y Groat, 1985; Friedrichsen y Dana, 2003), conocida en castellano como clasificación múltiple de ítems (CMI), la cual se fundamenta en la clasificación libre que hace el entrevistado de tarjetas, fotografías u objetos que conforman un dominio de interés para el investigador.
La principal ventaja de este modelo de entrevista está en que permite el surgimiento libre y espontáneo de categorías de los participantes para realizar clasificaciones sobre los elementos que se les entrega, en este caso las tarjetas con las situaciones temidas, buscando con ello que el investigador interfiera lo menos posible con sus preconcepciones en dichas clasificaciones, lo que suele ocurrir con las preguntas cuando se hace uso de cuestionarios.

La inclusión de la tarjeta "miedo" obedeció al interés de explorar las asociaciones entre las distintas situaciones con el temor. La tabla 1 describe las 23 situaciones que se asocian de manera más frecuente al miedo en la ciudad, más la tarjeta “miedo", conformando así el dominio del estudio.

Tabla 1.

Miedos en la ciudad

\begin{tabular}{llll}
\hline Robo en transporte público & 1 & Sitios desconocidos & 2 \\
\hline Paseo millonario en taxi & 3 & Ataques con sustancias & 4 \\
\hline Actos terroristas & 5 & Sufrir accidentes de tránsito & 6 \\
\hline Miedo & 7 & Violación & 8 \\
\hline Los desconocidos & 9 & Lugares solitarios y oscuros & 10 \\
\hline Los indigentes & 11 & Peleas callejeras & 12 \\
\hline Tribus urbanas & 13 & Los barristas & 14 \\
\hline Multitudes & 15 & Las epidemias & 16 \\
\hline Caños y humedales & 17 & Catástrofe natural & 18 \\
\hline Las palomas & 19 & Centro de la ciudad & 20 \\
\hline Asaltos y robos & 21 & Puentes peatonales & 22 \\
\hline Mimos y payasos & 23 & Marchas y disturbios & 24 \\
\hline
\end{tabular}

Fuente: elaboración propia.

\section{Instrucciones}

Para orientar la entrevista mediante la CMI, los participantes fueron entrevistados individualmente, y se les pidió que hicieran sus agrupaciones, a partir de las siguientes instrucciones:

Estamos haciendo un estudio acerca de lo que las personas piensan sobre algunas situaciones presentadas en estas 24 tarjetas, que corresponden a lo que las personas suelen temer cuando se movilizan por la ciudad. Por favor obsérvelas. Ahora, clasifíquelas en grupos, si- guiendo algún criterio, de manera que las tarjetas ubicadas dentro de cada grupo tengan algo similar o en común; además, cada grupo de tarjetas o situaciones debe diferenciarse de los otros grupos y llevar un nombre distinto. Usted puede elegir cualquier criterio que quiera para agrupar las tarjetas y puede dividirlas en tantos grupos cuantos quiera. No hay tipo alguno de clasificación correcta o incorrecta para sus respuestas, lo que cuenta es su opinión. Tómese el tiempo necesario y siéntase en libertad de hacer los comentarios que quiera. 


\section{Procedimiento}

Se partió de la entrevista efectuada a 50 personas, a quienes se les solicitó que respondieran de forma espontánea a la pregunta: ¿A qué le tiene miedo en la ciudad? Sobre la base de sus respuestas, se identificaron 23 situaciones, las cuales fueron presentadas en tarjetas, incluyendo una más con la palabra "miedo", para completar un total de 24 tarjetas.

Siguiendo el modelo de entrevista de clasificación múltiple de ítems, se entrevistó de manera individual a 80 personas -40 hombres y 40 mujeres-, a quienes, después de firmar un consentimiento informado y de confidencialidad, se les pidió que agruparan las tarjetas siguiendo sus propios criterios y dando lugar a sub-agrupaciones dentro de cada criterio. Esta operación se realizó dos veces con cada entrevistado.

La información recogida durante el primer semestre de 2013 se sistematizó en matrices, en las que se incluyeron, en las filas, las situaciones expuestas en las tarjetas, y, en las columnas, las distintas clasificaciones que hicieron los participantes. A cada clasificación se le asignó un número de forma secuencial, al igual que para cada subgrupo de tarjetas que iba creando cada participante. Las matrices se llenaron con los números del subgrupo en que cada participante las iba agrupando, según el criterio de agrupación. De este modo, se conformaron dos matrices para sistematizar la información de las clasificaciones libres que hicieron los hombres y las mujeres.

La información así sistematizada se analizó mediante el programa PERMAP, que analiza la información mediante un escalamiento multidimensional (MDS, por sus siglas en inglés), el cual correlaciona las variables bajo estudio y representa el grado de asociación de las variables en un mapa o gráfico de dos dimensiones, en el que la distancia espacial entre una y otra variable refleja el grado de relación entre ellas, al igual que el grado de asociación conceptual que establecen los participantes entre una y otra variable. El fundamento principal de este programa es que permite descubrir estructuras internas que pueden estar inmersas en un grupo de datos complejos. Ejemplos de la utilización de este tipo de entrevistas y análisis de la información mediante el escalamiento multidimensional aplicado al estudio del miedo pueden observarse en los trabajos de Roazzi, Federicci y Wilson sobre los miedos de los niños (2001); en estudios de criminalística para la captura de criminales en serie (Canter, 1994); y en la interpretación por parte de las personas sobre la guerra contra el terrorismo (Gray y Wilson, 2006).

A partir de la relación espacial que arrojó el programa PERMAP para el análisis de las matrices construidas a partir de las entrevistas a hombres y mujeres sobre las situaciones temidas en la ciudad, los investigadores procedieron a crean zonas o regiones sobre los gráficos mediante trazos, con el fin de generar una interpretación con miras a dar sentido a las agrupaciones observadas.

\section{Resultados}

Las figuras 1 y 2 , corresponden a los resultados arrojados por el programa PERMAP, después de que los investigadores hicieron los trazos que agrupan los ítems o tarjetas con las situaciones temidas para cada grupo de participantes. A continuación se describen los resultados para cada condición explorada en el estudio.

Clasificaciones para los hombres: La figura 1 es el resultado de la distribución arrojada por el programa a partir de las correlaciones entre las situaciones presentadas en las tarjetas según el subgrupo en que fueron agrupadas, y de los trazos que hicieron los investigadores a partir de las distancias espaciales entre las distintas situaciones bajo análisis. Se observa una distribución en la que en el centro aparece la tarjeta "miedo" y una distribución de las situaciones en cuatro regiones. Siguiendo las manecillas del reloj, se observa en la parte superior de la figura una agrupación que recoge lugares asociados con el temor, por cuanto las situaciones que allí se agrupan se refieren a la condición espacial que se asocia con los temores: puentes peatonales, caños y humedales, sitios desconocidos, etc.; en la segunda agrupación se 
observan las situaciones que involucran personas o animales asociadas con el miedo: payasos, tribus urbanas, marchas y disturbios, palomas; en la tercera se agrupan las situaciones impredecibles y que podrían estar fuera del control de los individuos: accidentes de tránsito, catástrofes naturales, etc,; y en la cuarta región se ubican los peligros que representan una afectación directa a la integridad o a los bienes del individuo: robos, ataques son ácido, paseos millonarios, etc.

Clasificaciones para las mujeres: La figura 2 muestra los resultados observados para el grupo de mujeres, donde, al igual que en el caso de los hombres, se pudieron crear regiones que agrupan las situaciones temidas. La información arrojada por el programa PERMAP dio lugar en este grupo a las mismas cuatro regiones que se pudieron crear para los participantes hombres; la primera, siguiendo las manecillas del reloj, agrupa las tarjetas o situaciones que involucran personas, multitudes o animales: barristas, palomas, indigentes, mimos, multitudes, etc; en la siguiente región se observan las situaciones sobre las cuales las personas piensan que es difícil o imposible ejer-

Figura 1. Estructura de los miedos en los hombres

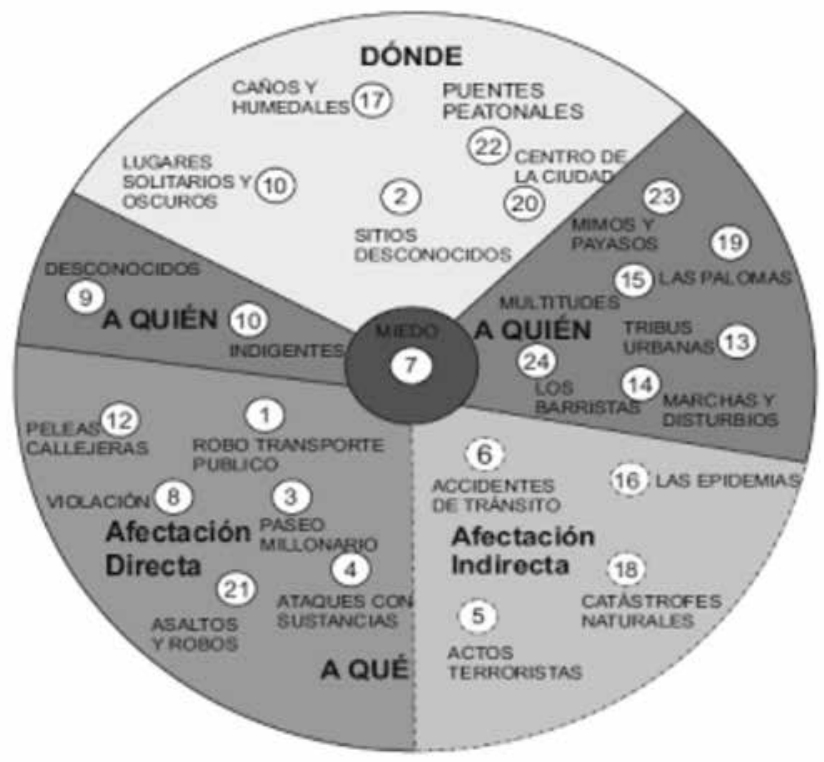

Fuente: elaboración propia. cer control: los actos terroristas, las epidemias o los accidentes de tránsito; en la tercera región se pueden observar las situaciones que involucran una afectación directa a la integridad personal, como el paseo millonario, los ataques con ácido o el robo. Por último se observan las situaciones que se asocian a lugares de miedo: puentes petatonales, caños y humedales, etc. A diferencia de lo que se observa en grupo de hombres, la tarjeta "miedo" en el segundo grupo se ubica dentro de la región de los ataques a la integridad personal.

\section{Conclusiones y análisis}

El miedo ha acompañado a la humanidad en el transcurso de su evolución, haciendo parte de su acervo filogenético y representando todavía un valor adaptativo. El miedo a lo que repta, a lo desconocido, a las alturas, a la oscuridad, está en nuestra filogénesis, pero también es producto de nuestra cultura, por las experiencias pasadas con las guerras, los desastres naturales, las epidemias; por haber sido víctimas de crímenes, o por la manera como los medios se encargan de gene-

Figura 2. Estructura de los miedos en las mujeres.

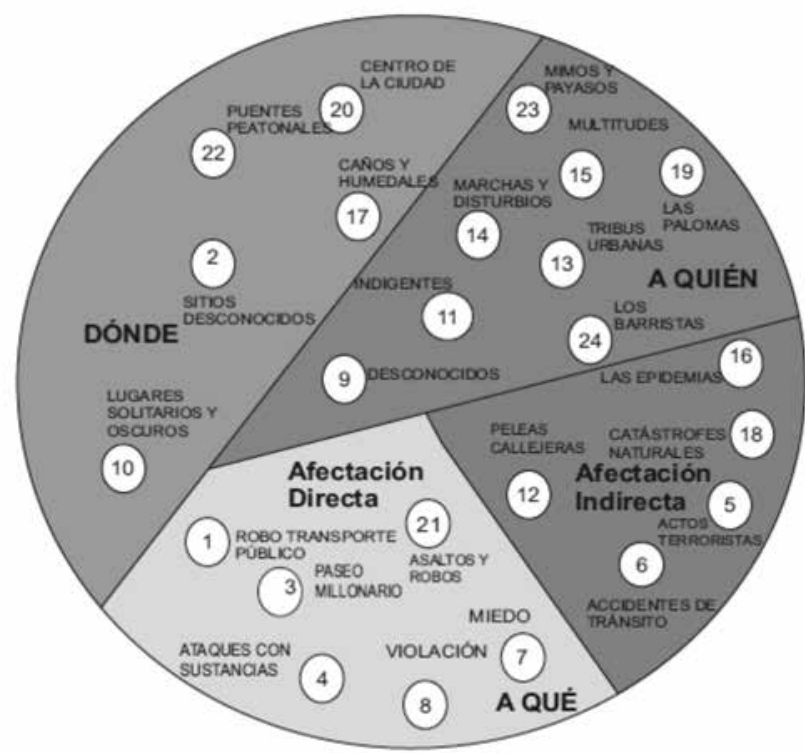

Fuente: elaboración propia. 
rarlo. Los temores se reflejan en la manera como los seres humanos construyen sus viviendas y ciudades, en los mitos y leyendas urbanas, y en la manera de comportarnos en el ambiente urbano.

Los resultados de las entrevistas llevadas a cabo en este estudio muestran que los miedos en la ciudad no se organizan de manera azarosa, sino que los individuos los organizan siguiendo una estructura conceptual, la cual se refleja en la manera como organizan las tarjetas y de la cual se valen, probablemente, para movilizarse por la ciudad y poder enfrentar situaciones que perciben como peligrosas.

Dentro de los resultados arrojados por el programa para computador PERMAP, a través de las gráficas, se ha podido develar que el miedo se organiza a partir de una estructura conceptual que identifica "lo temido", con afectación directa (atraco, violación, ataque con sustancias, etc.) o indirecta sobre el individuo (pestes, accidentes, etc.); los "lugares" asociados al miedo; y los "agentes" del miedo.

Si bien las 23 situaciones expuestas no abarcan todos los posibles miedos que puede tener una persona al transitar por la ciudad, los resultados reflejan principalmente la tipología de los temores. Cualquier otro no incluido en la identificación inicial de los que se tuvieron en cuenta en la fase inicial del estudio podría caber dentro del sistema conceptual que se logró reconocer en este estudio. Así, el temor a los parques o calles se ubicaría con seguridad en la sección correspondiente a los lugares, o el temor a ser rociado con ácido en la cara se ubicaría en el área de afectación directa, de tal suerte que los hallazgos permiten predecir en cierto grado dónde se ubicaría cualquier otro temor en el sistema conceptual del individuo.

Este tipo de hallazgos podrían ser de interés para las instituciones gubernamentales que quieren romper la discrepancia entre los datos objetivos de reducción de criminalidad (reducción de tasas de homicidios y de robos callejeros denunciados) y la percepción subjetiva de inseguridad, también conocida como mitos de inseguridad (Policía Nacional, 2010).
Sin dejar de reconocer que el miedo responde a factores objetivos, es posible hipotetizar que también resulta de procesos psicológicos más complejos, como la percepción de la ausencia de control sobre el ambiente (Páramo, 2004), evidenciado en estudios sobre hacinamiento (Baum y Paulus, 1987) y en la decisión de salir a caminar o a hacer ejercicio fuera de la casa (Giles-Corti y Donovan, 2002). En consecuencia, sería conveniente aumentar la percepción de control ambiental que pueda tener un ciudadano cuando circula por la ciudad, si la administración de la ciudad o la Policía actuaran de manera más eficiente al comunicar a la ciudadanía los resultados en la reducción de la criminalidad.

La información que suministren las autoridades encargadas de la seguridad deberán mostrar las estadísticas sobre criminalidad y educar al ciudadano en su interpretación al hacer énfasis en la reducción de los delitos sobre los cuales se tiene más temor y asegurarle, por ejemplo, que las acciones multitudinarias (barristas, tribus urbanas) no son agresivas, o que están controladas, y que la ciudad está preparada para atender eventualidades naturales y de salud (epidemias o catástrofes naturales).

Por otra parte, la administración de la ciudad deberá mejorar las condiciones de aquellos lugares que suelen asociarse con inseguridad al intervenir los espacios públicos con mobiliario urbano, luminarias, cámaras de seguridad, zonas verdes, áreas peatonales y ciclorutas; al mantener estos espacios limpios y promover actividades para que las personas se apropien de estos lugares, con lo cual se aumenta el grado de control percibido sobre el ambiente.

La teoría de conducta planeada (Ajzen, 1991; 2005; Ajzen y Fishbein, 2000) ha demostrado su valor para predecir comportamientos de diverso tipo a partir de tres factores: la actitud hacia la conducta en cuestión (determinada por valores y creencias), las normas sociales y el control percibido que se tenga sobre la conducta por ejecutar - para el caso que interesa, salir a la calle-.

En apoyo a la disposición del individuo a salir a la calle, los medios juegan un papel preponderante 
al momento en que la ciudadanía emita un concepto favorable con relación a la percepción de seguridad. Es importante buscar su compromiso con el aumento de información referente a la seguridad, y no solo a las afectaciones que se tienen a diario por la delincuencia en general, la cual es, en los últimos tiempos, el foco de atención de los noticieros de televisión en las mañanas. Estos deberían involucrarse de manera más amplia en la difusión de los logros, las acciones y otras actividades que las administraciones adelanten contra el crimen en la ciudad, con el fin de minimizar la percepción de inseguridad que afecta a la ciudadanía. Si se lograra ampliar de forma directa este discurso, permitirá afianzar la confianza de los ciudadanos en sus administraciones y en la Policía, en la medida en que muestren que estas instituciones están ejerciendo las medidas apropiadas para mejorar la seguridad y, con ello, lograr una mayor apropiación de los espacios públicos y un mejoramiento de la calidad de vida urbana.

Por otra parte, la integración y participación entre los distintos sectores de la sociedad que hacen uso de los espacios públicos y los profesionales que desarrollan y diseñan estos espacios permitirá que tanto la sociedad como los entes gubernamentales lleguen a un consenso que proporcione no solo un diseño y uso adecuado de dichos espacios, sino también una mayor identidad de los lugares públicos de la ciudad. Las ciudades con sus gobernantes deberán gestionar mecanismos que permitan generar un sentimiento de pertenencia de los ciudadanos hacia su sector, logrando una identificación del sitio en el que viven y, con ello, estructurar un tejido capaz de fomentar acciones que lleven a una cohesión social.

La Policía, como entidad gubernamental encargada de mantener el orden público y la seguridad de los ciudadanos, deberá diseñar políticas que se encaminen a fortalecer los mecanismos de participación, como la denuncia del delito, y, con ello, la percepción de control de los ciudadanos sobre el ambiente..

Este trabajo hace un aporte interesante al estudio del miedo en las ciudades, no solo al develar la estructura conceptual de sus habitantes sino también al introducir una técnica de recolección de información de tipo cualitativo para este tipo de fenómenos urbanos, los cuales, generalmente, son explorados mediante la aplicación de cuestionarios, que si bien establecen generalizaciones, no permiten comprender muchas veces la complejidad de los fenómenos que estudian.

Como aporte final de este trabajo, y para futuras investigaciones, será necesario involucrar otros sectores de la población que identifiquen diferentes situaciones temidas y explorar más a fondo cómo cambiar las percepciones del temor como resultado de la investigación a partir de la gestión que adelanten las instituciones gubernamentales y las asociaciones civiles.

\section{Referencias}

Ajzen, I. (1991). The theory of planned behavior. Organizational Behavior of Human Decision Processes, 50(2), 179-211.

Ajzen, I. (2005). Attitudes, personality and behavior. Maidenhead: McGraw-Hill.

Ajzen, I., \& Fishbein, M. (2000). Attitudes and the attitude-behavior relation: Reasoned and automatic processes. En: W. Stroebe \& $M$. Heawstone (Eds.), European Review of Social Psychology, pp. 1-33.

Altheide, D. L., \& Gruiter, A. (2002). Creating fear: News and the construction of crisis. Canadian Journal of Sociology Online, Recuperado de http://www.cjsonline.ca/pdf/fear.pdf

Baum, A., \& Paulus, P. (1987). Crowding. En D. Stokols \& I. Altman (Eds.), Handbook of Environmental Psychology (533-570).). Nueva York: Wiley.

Bocanegra, D. F., y Nieto, D. L. E. (2010). Modelos de atención a víctimas de delitos: Revisión conceptual y observación de la experiencia de tres centros de victimoasistencia en la ciudad de Bogotá. Diversitas: Perspectivas en Psicología, 6(2), 321-338. 
Bogotá Cómo Vamos. (2013). Recuperado de http://www.bogotacomovamos.org/documentos/ \#percepcion+page__1

Borja, J. (2005). Urbanismo y ciudadanía: Civismo; Las claves de la convivencia. Barcelona.

Borja, J. (2008, enero-febrero). Miedos, segregación y mercado en la ciudad globalizada. $R e$ vista Nueva Sociedad, p. 213.

Bru, J., y Vicente, J. (2005). Qué produce miedo en la ciudad. vII Coloquio de Geografía Urbana, Girona, España. Girona: Universitat de Girona, pp: 15-28

Condon, S., Lieber, M., \& Maillochon, F. (2007). Feeling unsafe in public places: Understanding women's fears. Revue Francaise de Sociologie, 5(48), 101-128.

Cámara de Comercio de Bogotá. (2013). Encuesta de percepción y victimización. Recuperado de http://www.ccb.org.co/contenido/contenido. aspx?cat ID $=126 \&$ con $I D=562$

Canter, D. (1994). Criminal shadows: Inside the mind of the serial killer. Glasgow: Harper Collins.

Canter, D., Brown, J., \& Groat, L. A. (1985). Multiple Sorting Procedure. En M. Brenner, J. Brown \& D. Canter (Eds.), The research interview: Uses and approaches. Londres: Academic Press.

Carrión, F. (2007). Percepción inseguridad ciudadana. Extraído el 28 de abril de 2008 de: http://www.flacso.org.ec/docs/ciudad_segura15.pdf

Carro, D., Valera, S., \& Vidal, T. (2010). Perceived insecurity in the public space: Personal, social and environmental variables. Qual Quant, (44), 303-314.

Cuevas, E., y Gómez, S. Y. (2014, junio). En busca de la seguridad en el espacio público: Aproximaciones urbanas de Xalapa, México. Papeles de Coyuntura (38), 46-58.
Farrall, S. (2007). Experience and expression in the fear of crime.. Swindon: Economic and Social Research Council.

Ferraro, K., \& Grange, L. (1987). The measurement of fear of crime. Sociological Inquiry, $57(1)$.

Fraile, P., Bonastra, Q., Rodríguez, G., y Arella, C. (2010). Seguridad, temores y paisaje urbano. Barcelona: Ediciones del Serbal.

Friedrichsen, P. M., \& Dana, T.M. (2003). Using a card-sorting task to elicit and clarify scienceteaching orientations. Journal of Science Teacher Education, 14(4). 291-309.

Gallup. (2013). Latin America scores lowest on security. Recuperado el 21 de agosto de 2014 de http://www.gallup.com/poll/175082/latinamerica-scores-lowest-security.aspx?.

Giles-Corti, B., \& Donovan, R. J. (2002a). The relative influence of individual, social, and physical environment determinants of physical activity. Social Science \& Medicine, 54(12), 1793-1812.

Gray, J. M., \& Wilson, M. A. (2006). Understanding the "War on Terrorism": Responses to 11 September 2001. Journal of Peace Research, 43(1), 23-36.

Gutiérrez, O. (Coord.). (2005). La ciudad y el miedo. vII Coloquio de Geografía Urbana, . Girona: Universitat de Girona

Hale, C. (1996, January). Fear of crime: A review of the literature. International Review of Victimology, 4(2), 79-150.

Hawdon, J., Räsänen, P., Okasen, A., \& Vuori, M. (2013). Social responses to collective crime: Assessing the relationship between crime-related fears and collective sentiments. European Journal of Criminology, 0(0), 1-18.

Hackett, P. M. W. (2014). Facet theory and mapping sentence: Evolving philosophy, use and application. Nueva York: Palgrave Macmillan. 
Jackson, J. (2002). The fear of crime: An analysis and development of theory and method. Tesis de doctorado inédita, University of London, London School of Economics and Political Science, Londres.

Kelly, G. (1991). The psychology of personal constructs: Theory and personality (Vol. 1). Londres: Routledge Publishers.

León, M. E. (2008). El miedo y la percepción ciudadana en las políticas de seguridad ciudadana en Bogotá (Trabajo de grado, Pontificia Universidad Javeriana, Bogotá).

Linke, U., \& Smith, D. (2009). Cultures of fear. Nueva York: Pluto Press.

Macek, S. (2006). Urban nightmares: The media, the right, and the moral panic over the city. Minneapolis: Minessota Press.

Manrique, D. J. (2006). Aplicación de la entrevista cognoscitiva y de la entrevista judicial a un testigo víctima del delito de hurto. Diversitas: Perspectivas en Psicología, 2(1), 55-70.

Morquecho, A. C., y Vizcarra, L. R. (2008, abril). Inseguridad pública y miedo al delito: Un análisis de las principales perspectivas teóricas y metodológicas para su estudio. Letras Jurídicas, (6), 1- 20.

Murphy, G. L. (2002). The big book of concepts. Cambridge: The MIT Press.

Niño, S. (2007). Territorios del miedo en Santa Fe de Bogotá. Bogotá: Tercer Mundo.

Páramo, P (2011). Sociolugares. Bogotá. Universidad Piloto de Colombia

Páramo, P. (2014). El miedo a la ciudad. En A. Burbano y P. Páramo (Eds.), La ciudad habitable: Espacio público y sociedad. Bogotá. Universidad Piloto de Colombia.

Páramo, P., y Cuervo, M. (2009). La experiencia urbana en el espacio público de Bogotá en el siglo xx. Bogotá: Universidad Pedagógica Na-
cional/Universidad Santo Tomás/ Universidad Piloto de Colombia.

Páramo, P., \& Cuervo, M. (2013). Historia social situada en el espacio público de Bogotá desde su fundación hasta el siglo XIX. Bogotá: Universidad Pedagógica Nacional.

Photos, E. M., \& Willis, A. J. (Eds.). (2011). Formal approaches in categorization. Cambridge: Cambridge University Press.

Policía Nacional. (2010). Estrategia de la Policía Nacional para la consolidación de la seguridad ciudadana. Bogotá: Policía Nacional.

Portal, M., y Díaz, R. (2001). Vivir la diversidad: Identidades y cultura en dos contextos urbanos de México. Alteridades, 11(22),

Roazzi, A., Federicci, F., y Wilson, M. (2001). A estructura primitiva da representacao social do medo. Psicologia: Reflesao e Crítica, 14, 57-72.

Russo, S., Roccato, M., \& Vieno, A. (2013). Criminal victimization and crime risk perception: A multilevel longitudinal study. Social Indicators Research, 112(3), 535-548.

Soyinka, W. (2007). Clima de miedo. Barcelona: Tusquets.

Valentine, G. (1989). The geography of women's fear. Area, 21(4), 385-390.

Varela, S., Castrechini, A., \& Di Masso, A. (2013). Perceived insecurity in the public space: Press analysis study. En 22 IAPS CONFERENCE: Human experience in the natural and built environment; Implications for research policy \& practice, 278.

http://ccb.org.co/contenido/contenido.aspx?cat ID $=126 \&$ con ID $=6574$

http://www.bogotacomovamos.org/documentos/ presentacion-de-resultados-comparados-dela-encues/

http://www.actionaid.org/sites/files/actionaid/ women_and_the_city_ii_1.pdf 